\title{
Treatment of late benign post-esophagectomy tracheal- gastric fistula with tracheal stenting
}

\author{
Aaron Sparks, Trevor Beckham and James R Stewart* \\ University of Missouri-Kansas City, School of Medicine, Truman Medical Center, Department of Surgery, Kansas City, Missouri, USA
}

\begin{abstract}
We present a case of late benign tracheal-gastric fistula treated with a covered tracheal stent. The patient had a previous esophagectomy for cancer. He developed a tracheal-gastric fistula 14 months later after a traumatic intubation during repair of an abdominal incisional hernia. The patient did not want another major operation. We treated the patient with a covered tracheal stent and he had complete resolution of symptoms. We believe this is the first case report of Ultraflex ${ }^{\circledR}$ stent placement for a tracheal-gastric fistula.
\end{abstract}

\section{Introduction}

Tracheal-gastric fistula is a rare complication after esophagectomy. The majority occur early in the postoperative period [1]. Several etiologies exist including: anastomotic leak with tracheal erosion, tracheal ischemia, cuff-induced tracheal necrosis, and secondary to dilation of anastomotic stenosis [1]. We present a patient with iatrogenic tracheal-gastric fistula after traumatic intubation. It presented in a delayed fashion 14 month after the initial esophagectomy. The patient was treated with a covered Ultraflex tracheal stent. A review of the literature primarily yields case reports and small case series discussing tracheal-gastric fistulas related to complications of esophagectomy. We believe this is the first report of tracheal stenting with a covered tracheal stent for a benign, late tracheal-gastric fistula.

\section{Case report}

J.C. was diagnosed with clinical stage IIIB esophageal adenocarcinoma. He received neoadjuvant chemotherapy and subsequent transhiatal esophagectomy twenty months earlier, at an outside institution. Postoperatively the patient required multiple anastomotic dilatations and eventual esophageal stent placement for anastomotic stenosis. The stent was subsequently removed with resolution of dysphagia. The patient developed a midline abdominal incisional hernia which was repaired 14 months after the esophagectomy. The post-operative course was complicated by a persistent cough. Symptoms were significantly exacerbated by drinking liquids. The patient was referred to Thoracic Surgery Clinic nine months after the successful hernia repair. A video swallow demonstrated a trachealgastric fistula. The diagnosis was confirmed via CT scan (Figure 1), and contrasted CT in the prone position. Treatment options including tracheal resection with repair of the gastric fistula were discussed with the patient. However, the patient did not want an additional major operative procedure. He was symptomatic and needed intervention to prevent recurrent aspiration pneumonia, which he had experienced. The patient did agree to tracheal stenting. A covered $18 \mathrm{~mm} \times 40 \mathrm{~mm}$ Ultraflex ${ }^{\oplus}$ stent was placed using rigid bronchoscopy and fluoroscopic guidance.
The patient had complete resolution of his aspiration symptoms. The patient tolerated a diet without any coughing. A follow-up CT scan showed no communication between the trachea and intrathoracic stomach. After 3 months the patient desired to have the stent removed. After removal with rigid bronchoscopy, he had almost immediate return of aspiration symptoms and imaging showed a persistent communication. The patient's $18 \mathrm{~mm} \times 40 \mathrm{~mm}$ Ultraflex ${ }^{\oplus}$ covered tracheal stent was replaced and he remains asymptomatic 12 months later (Figure 2).

\section{Discussion}

Tracheal-gastric fistula is a rare complication after esophagectomy. Iatrogenic tracheal injury has a reported incidence of $0.05-0.37 \%$ [2]. The literature consists of case reports and small case series. No large series have been reported. Chest X-ray is often the first diagnostic modality requested, followed by CT scan and visualization with bronchoscopy, the gold standard [2]. Treatment options regarding tracheal-gastric fistula include both operative and non-operative approaches. We treated our patient with a covered, flexible endotracheal stent. Airway stenting, using an endoscopic approach, has been used for proximal tracheobronchial malignancy, head and neck tumors, tracheal stenosis, tracheobronchial malacia and vascular compromise [3]. The patient's disease process determines the treatment modality. Patients benefiting from stent placement include patients with high surgical risk, patients refusing surgery, or patients with a disease process not amenable to surgery [3]. Both silicone and metal stents are utilized for tracheal stenting. Silicone stents allow for easy repositioning and removal $[3,4]$ but would not ideal for a fistula. The stent used in our case was a covered Ultraflex ${ }^{\oplus}$ stent, sized according to the imaging studies. It is

Correspondence to: James R. Stewart, MD, University of Missouri-Kansas City, School of Medicine, Department of Surgery, 2301 Holmes Street, Kansas City, Missouri 64108, USA, Tel: 816-404-5372; Fax: 816-404-5381 E-mail: jrs1040@ aol.com

Key words: Tracheal-gastric fistula, esophagectomy complications, airway stenting Received: March 24, 2018; Accepted: April 18, 2018; Published: April 21, 2018 


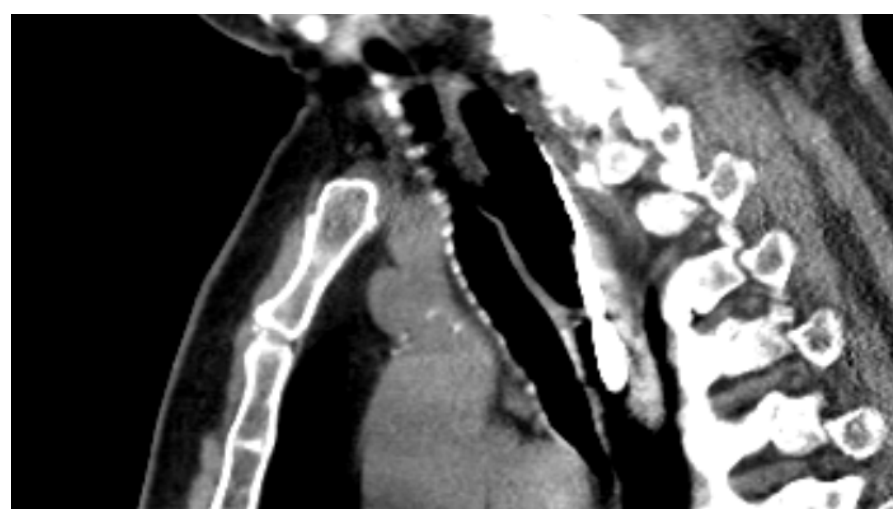

Figure 1. Sagittal cut of CT scan demonstrating $11 \mathrm{~mm}$ tracheal gastric fistula.

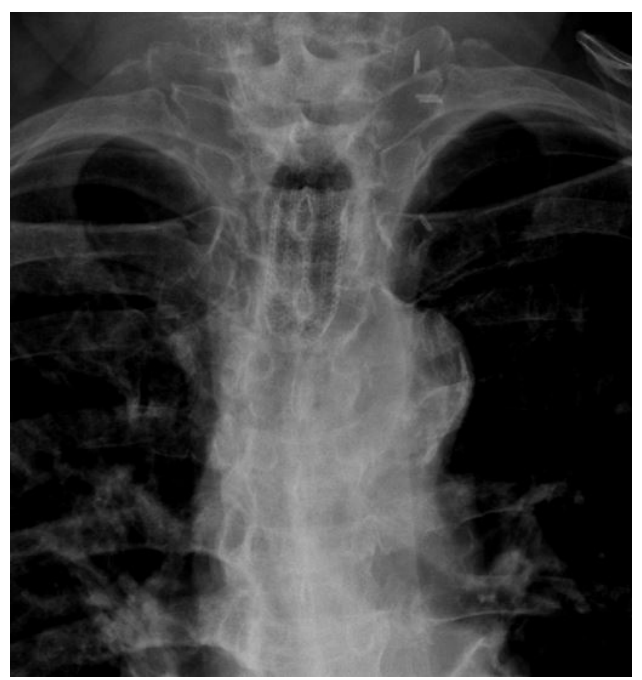

Figure 2. Radiograph demonstrating stent in mid-trachea. single strand woven nitinol covered with silicone. In our judgement, placement of a gastric stent would not have been effective in sealing the fistulous communication.

\section{Conclusion}

Tracheal gastric fistula is a rare complication after esophagectomy. We describe the successful treatment of a benign, late postesophagectomy tracheal-gastric fistula with a flexible silicone covered endotracheal stent. It is a novel approach and was tailored to treat a patient who did not want additional surgery. At 12-month follow-up the patient remains asymptomatic. We believe this is the first case report of Ultraflex ${ }^{\oplus}$ stent placement for this condition.

\section{References}

1. Buskens CJ, Hulscher JBF, Fockens P, Obertop H, van Lanschot JJB (2001) Benign tracheo-neo-esophageal fistulas after subtotal esophagectomy. Ann Thoracic Surg 72 : 221-224. [Crossref]

2. Tazi-Mezalek R, Musani AI, Laroumagne S, Astoul PJ, D'Journo XB, et. al. (2016) Airway stenting in the management of iatrogenic tracheal injuries: 10-year experience. Respirology 8: 1452-1456. [Crossref]

3. Airway Stenting. Available at https://www.ctsnet.org/article/airway-stenting Accessed July 28, 2017.

4. Wood, DE (2004) Tracheal and bronchial stenting. In: Grillo HC, ed. Surgery of the Tracheal and Bronchi. Ontario, Canada: BC Decker.40: 763-790.

Copyright: $\odot 2018$ Sparks A. This is an open-access article distributed under the terms of the Creative Commons Attribution License, which permits unrestricted use, distribution, and reproduction in any medium, provided the original author and source are credited. 